Antonia M. Molinari, OMS III and Jay H. Shubrook*, DO

\title{
Treatment options and current guidelines of care for pediatric type 2 diabetes patients: a narrative review
}

https://doi.org/10.1515/jom-2020-0172

Received July 3, 2020; accepted November 3, 2020;

published online February 19, 2021

\section{Abstract}

Context: Type 2 diabetes (T2D) mellitus, which was once considered a disease affecting adults, is a growing problem among youths. Research now shows that T2D in youths is more progressive and associated with earlier onset of complications than adult-onset T2D.

Objectives: To update practicing clinical physicians on what is currently known about pediatric T2D and share current guidelines to care for these patients.

Methods: The authors conducted a literature review through the MEDLINE/PubMed, Google Scholar, and clinicaltrials.gov databases in July 2019 using the following search phrases: "youth-onset type 2 diabetes," "pediatric type 2 diabetes," "diabetes in youth," and "FDA-approved pediatric diabetes medications.” Items published between 2010 and 2019 and written in English were included.

Results: A total of 65 items were included after database review, including 44 articles, 10 FDA product inserts, six clinicaltrials.gov listings, three position statements/guideline documents, one website, and one FDA announcement. Conclusions: There are very limited treatment options available to manage pediatric T2D. Prevention of pediatric T2D is paramount, which requires a high index of suspicion, routine screening of children and adolescents, and willingness to engage in family-based interventions for those at risk for prediabetes and T2D. Osteopathic physicians have a unique opportunity to help by applying the osteopathic principles to the whole person - including

*Corresponding author: Jay H. Shubrook, DO, College of Osteopathic Medicine, Touro University California, 1310 Club Drive, Vallejo, CA 94592-1187, USA, E-mail: jay.shubrook@tu.edu

Antonia M. Molinari, OMS III, College of Osteopathic Medicine, Touro University California, Vallejo, CA, USA environmental, societal, and lifestyle factors - to help patients achieve their health and wellness goals.

Keywords: diabetes treatment; obesity; pediatrics; type 2 diabetes.

Type 2 diabetes (T2D) mellitus has long been considered a disease affecting adults, but now about $24 \%$ of children diagnosed with diabetes have T2D [1]. The estimated annual increase in incidence of T2D among youths was $4.8 \%$ based on incident rates from 2002 to 2012 [2], and research suggests that prevalence continues to grow rapidly [1].

T2D diagnosed in youth is more progressive and aggressive than adult-onset T2D [3-5]. T2D among youths also appears to be more resistant to the standard treatment options, including therapeutic lifestyle changes, and complications develop more quickly in children than adults [6-8]. Furthermore, there are fewer FDA-approved pharmacologic options for pediatric patients [6, 7]. Among other key risk factors, childhood obesity predisposes youths to an insulin-resistant state and, when compounded with insulin-resistance during puberty, places overweight pediatric patients at risk [9]. The best chance at slowing the epidemic is for physicians to become familiar at identifying at-risk youths and providing early intervention that emphasizes family-based preventive lifestyle modifications within pediatric populations.

The purpose of this narrative review is to update practicing clinical physicians on what is presently known about pediatric patients with T2D and to educate clinicians on the current guidelines of care for these patients.

\section{Methods}

The authors conducted a literature review in July 2019 focused on pediatric T2D by searching PubMed, Google Scholar, and clinicaltrials.gov with the following phrases: "youth-onset type 2 diabetes," "pediatric type 2 diabetes," "diabetes in youth," and "FDA-approved pediatric diabetes medications.” Resources published between 2010 and 2019 and written in English were eligible for inclusion. The reference lists of the articles found in the initial search were reviewed for additional 
material and specific FDA medication package insert were also included. Both authors reviewed all resources and mutually determined which to include in the final review.

\section{Results}

A total of 65 items were included and analyzed after the authors collaboratively reviewed the search results, including 44 articles, 10 FDA product inserts, six clinicaltrials.gov listings, three position statements/guideline documents, one website, and one FDA announcement. In the narrative review presented in the Discussion, we summarize these findings in these resources in a detailed overview of pediatric T2D, including what makes it distinctive, how to diagnose it, and what treatment options exist.

\section{Discussion}

\section{Pathophysiology}

Pediatric patients are not metabolically identical to adults with regard to T2D. The Restoring Insulin Secretion Study (RISE) [3, 4] found that youths, compared to adults, have lower insulin sensitivity, hyper-responsive $\beta$-cells, decreased insulin clearance by the liver, and more rapid decline in $\beta$-cell function. Puberty alone is associated with lower insulin sensitivity, which the body adjusts for by increasing insulin secretion [9]. During puberty, peripheral insulin sensitivity decreases due to an increase of hormonal changes like testosterone, growth hormone, and estrogen [10].

Obesity has also been identified as an insulin-resistant state [11]. The prevalence of childhood obesity was $18.5 \%$ in 2015-2016 and has continued to rise [12]. The increase in visceral fat associated with obesity is hypothesized to increase peripheral insulin resistance in various ways. Visceral fat secretes adipokines that alter the responsiveness of target tissues to insulin, effecting the signaling cascasde [13]. Obesity is also associated with increased free fatty acids and lipids circulating in the body that can accumulate and induce lipotoxicity in tissues, resulting in insulin resistance [13]. In adolescents during puberty, there is the hormone-induced decrease in insulin sensitivity and the insulin resistance associated with obesity that overload the dynamic response of $\beta$-cells. Together, these two major factors work synergistically to place peripubertal youth at an increased risk for T2D.

\section{Role of genetics}

A family history of T2D has long been identified as a prominent risk factor for developing T2D in adults [7]. In fact, more than 100 alleles are associated with T2D. In the Treatment Options for Type 2 Diabetes in Adolescents and Youth (TODAY) cohort [5], 89.4\% of pediatric participants had a first-degree relative or grandparent with T2D.

\section{Screening those at risk: who should we screen?}

The American Diabetes Association (ADA) recommends screening pediatric patients after they begin puberty or after they turn 10 years old if they have a body mass index (BMI) greater than or equal to the 85th percentile and at least one additional risk factor present [7]. Risk factors include [7]:

- Maternal history of diabetes or gestational diabetes during child's gestation.

- Family history of T2D in a first- or second-degree relative.

- Racial/ethnic heritages such as Native American, African American, Latino, Asian American, and Pacific Islander.

- Signs of insulin resistance (including conditions associated with): acanthosis nigricans, hypertension, dyslipidemia, polycystic ovary syndrome, or low-forgestational-age birth weight.

In the TODAY cohort [5], 85.6\% of participants exhibited signs at baseline of acanthosis nigricans on the neck. In the same cohort, $80.5 \%$ of participants presented with dyslipidemia at baseline, $13.6 \%$ with hypertension, and 33.3\% with a maternal history of gestational diabetes during child's gestation [5]. The composition of the cohort also included a higher proportion of minority groups, as demonstrated in Table 1 [5].

\section{Diagnosis}

Current diagnosis guidelines are based on the adult laboratory values for prediabetes and $\mathrm{T} 2 \mathrm{D}$, as seen in Table $2[7,14]$.

The ADA recommends repeat laboratory testing for confirmation unless initial presentation involves hyperglycemic crisis, including obtaining laboratory results testing for pancreatic autoantibodies to rule out type 1

Table 1: Ethnic distribution of type 2 diabetes in the TODAY cohort $(n=704)$, as selected from a predetermined list [5].

\begin{tabular}{lr}
\hline Self-reported ethnicity & TODAY cohort participants n (\%) \\
\hline Hispanic & $289(41.1 \%)$ \\
Non-Hispanic Black & $222(31.5 \%)$ \\
Non-Hispanic White & $138(19.6 \%)$ \\
American Indian & $43(6.1 \%)$ \\
Asian & $12(1.7 \%)$ \\
\hline
\end{tabular}


Table 2: Laboratory range values for diagnosis of prediabetes and diabetes from the 2020 ADA guidelines [7, 14].

\begin{tabular}{lll}
\hline $\begin{array}{l}\text { Glycemic } \\
\text { measure/test }\end{array}$ & $\begin{array}{l}\text { Prediabetes } \\
\text { range }\end{array}$ & Diabetes range $^{\text {a }}$ \\
\hline $\begin{array}{l}\text { Hemoglobin A1c\% } \\
\text { Fasting glucose }\end{array} \quad \geq 100$ to $<126 \mathrm{mg} / \mathrm{dL}$ & $\geq 126 \mathrm{mg} / \mathrm{dL}$ \\
OGTT - $2 \mathrm{~h}$ & $\geq 140 \rightarrow<200 \mathrm{mg} / \mathrm{dL}$ & $\geq 200 \mathrm{mg} / \mathrm{dL}$ \\
postprandial & \\
glucose & Random plasma glucose \\
\hline${ }^{\text {a } C r i t e r i a ~ f o r ~ d i a g n o s i s ~ d i a b e t e s ~}$ & $>200 \mathrm{mg} / \mathrm{dL}$, symptoms of \\
is confirmed with at least 1 & classic hyperglycemia, or \\
of the following. & hyperglycemic crisis \\
\hline
\end{tabular}

diabetes (T1D) [7]. Genetic testing, if monogenic diabetes is suspected, may also be indicated [7]. In the TODAY study group [15], 9.8\% of participants tested positive for either glutamic acid decarboxylase antibodies (GAD-65), islet antigen (IA-2) antibodies or both, and $9.8 \%$ were clinically diagnosed with T2D based on clinical presentation.

The validity of A1c, fasting glucose, and OGTT tests as diagnostic criteria in pediatric populations remain questionable [7]. As an indirect measure of glycemic control, hemoglobin A1c (HbA1c) values can be altered by certain medications, hemoglobinopathies (such as thalassemia or sickle cell), age, and variations in sensitivity between certain ethnic groups [16]. Benefits of using HbA1c to screen at-risk populations include not requiring fasting, having less variability, and being unaffected by external circumstances such as illness and stress at the time of blood draw [16]. However, current research suggests HbA1c alone is not a reliable tool to diagnosis prediabetes and T2D in pediatric patients due to evidence that it underestimates prevalence of dysglycemia in obese youths due to low sensitivity and specificity [17].

Fasting plasma glucose (FPG), 2-h postprandial glucose levels, and the post-oral glucose tolerance test (OGTT) have also not been significantly studied in youths, leaving diagnostic criteria based on cut-off values studied in adult-only populations, similar to HbA1c [16]. Other weaknesses of these tests include the stress and feasibility of children completing an 8-h fast overnight, meeting at least a 2-h time requirement in-office for OGTT, and an additional task for staff to properly administer in office [16]. In addition, FPG, OGTT, and HbA1c all look at different parts of glycemic control, suggesting a value for each in the role of diagnosis and why Barr in 2019 [16] recommended using FPG, OGTT, and HbA1c together to screen for pediatric T2D and prediabetes. For example, a higher OGTT identifies more postprandial insulin resistance while a higher FPG shows more hepatic insulin resistance [16]. Consequently, the ADA still recommends at least one laboratory test (Table 2) and repeated labs in the absence of overt hyperglycemic crisis. When laboratory testing is not clear or if waiting for diagnostic results, treating patients as if they have T1D instead of T2D remains the safer option.

\section{Socioeconomic and psychosocial factors}

The ADA recommends that healthcare providers consider possible food insecurity, housing instability, and financial limitations when developing a feasible and effective treatment plan for families and patients with T2D, including children [7].

Children with T2D are more likely to suffer from other mental health issues compared to children without chronic illness [18]. Stress, isolation, depression, anxiety, substance abuse, and eating disorders should be screened for by providers regularly in youth with T2D. Chronic exposure to stressors places these individuals at higher risk for developing psychosocial problems $[18,19]$. It can also interfere with how individuals manage their diabetes care, leading to poorer glycemic control [19]. In the TODAY cohort, $14.8 \%$ [20] of the 704 participants reported clinically significant symptoms associated with depressive disorders compared to $11.3 \%$ [21] estimated in the general adolescent population. Depressive symptoms were more prominent in older (age $\geq 16$ years) female participants, but generally consistent across the different races/ethnicities in that group [20]. In addition, $26 \%$ of the TODAY cohort participants reported some form of binge eating [22]. These same participants had higher rates of obesity and waist circumference, and reported lower quality of life ratings [22]. Thus, ADA guidelines recommend regularly screening for diabetes distress and mental health issues in pediatric patients with T2D [7].

\section{Prevention}

\section{Childhood obesity: how does this affect pediatric T2D?}

Preventing and treating childhood obesity is a powerful step toward avoiding the development of pediatric T2D in at-risk youth [23]. Childhood obesity is a complex problem that extends beyond the health behaviors of a child and should be considered a possible reflection of family, socioeconomic, and environmental factors. Obesity is often an insulin-resistant state and the most common comorbidity in pediatric T2D $[11,24]$. Obesity is defined as an age 
and gender specific BMI in the 95th percentile ( $z$-score, 1.645) or higher [25]. To put this into perspective, the mean BMI $z$-score in the TODAY study was 2.15, indicating a high prevalence of obesity in the cohort [20]. While there are many causes leading to both obesity and T2D, certain traits and habits can be useful in the early detection and prevention of childhood obesity. Specific risk factors providers can use to identify potential at-risk youth include "parental obesity, poor nutrition, low levels of physical activity, inadequate sleep, sedentary behaviors, and low family income" [25]. Weight loss improves insulin resistance and is a key component of management of T2D. Providers should begin to screen children early for the factors mentioned above. Further, when a child is found to have excessive weight, the physician should address it immediately and work with the family to treat obesity and/or prevent development of obesity-related chronic disease. Family-based interventions will need to account for possible socioeconomic factors and food insecurities when developing treatment plans.

\section{Prediabetes}

National Health and Nutrition Examination Survey (NHANES) cross-sectional studies published in 2018 [26] and 2020 [27] found that the rate of obesity in youth was $18.5 \%$ and prediabetes was found in $18 \%$ of adolescents.

For those identified with impaired glucose tolerance or prediabetes (Table 2; see also the Diagnosis section of this review), the best recommended initial treatment plan is intensive lifestyle interventions [28]. Currently, metformin and insulin are not recommended for youths with prediabetes based on evidence from the Restoring Insulin Secretion (RISE) trial [29]. In summary, physicians should identify those at risk, especially patients with obesity and/or prediabetes, and initiate integrative family-based lifestyle interventions early.

\section{Treatment}

Youth-onset T2D is more virulent and more progressive than adult-onset T2D [6], meaning that a gentle and progressiveintensity approach is less likely to be successful. Instead, treatment should start immediately upon diagnosis. Titration of treatment may need to be more aggressive and of shorter duration than in adults. All children should receive team-based family care and require close supervision and follow up.

\section{Treatment goals}

The ADA recommends initiating comprehensive lifestyle programs and diabetes education for families, with a goal of $7-10 \%$ decrease in body weight $[7,14]$. The glycemic target for most pediatric populations with T2D, according to the ADA, is an $\mathrm{HbA1c}<7 \%$ for patients taking only oral medications [7]. Lower A1c goals can be considered for individuals who have certain disease characteristics (e.g., if providers believe that a lower goal is achievable without causing hypoglycemic episodes) [7]. Every three months, pediatric patients with T2D should have their HbA1c checked, and treatment should be intensified if the HbA1c goal has not been achieved [14].

\section{Lifestyle interventions}

According to ADA guidelines, newly diagnosed children with T2D and their families should be counseled on lifestyle interventions and given diabetes education [14]. Recommendations focus on weight loss, exercise, behavioral changes, and diet modification. Children should be advised to get at least 30-60 min of moderate-to-vigorous physical activity at least five days per week and at least three days of strength training per week, in addition to reducing prolonged sedentary behaviors. The American Academy of Pediatrics (AAP) recommends less than $2 \mathrm{~h}$ of screen time per day not related to school work [28].

It is important to note a unique aspect regarding the implementation of lifestyle modifications for pediatric patients. These patients are usually not responsible for determining which foods are brought into the home or prepared, highlighting the need to engage the entire family when attempting to implement lifestyle changes. It is important to emphasize healthy eating habits with nutrient-rich, high-quality food and reduction of highcalorie, low-nutrient foods, especially sugar-added beverages $[7,14]$. Interventions should be based on a chronic care model with a goal of $7-10 \%$ decrease in body weight [14].

\section{Standard pharmacotherapy}

Metformin therapy has been used as a comparison therapy in previous trials, including TODAY [30] and RISE [4, 31]. In one of the first studies of metformin in pediatric T2D patients, metformin was determined to improve glycemic control in youths, with an average reduction in fasting blood glucose levels of $42.9 \mathrm{mg} / \mathrm{dL}$. While this trial had a small sample size (82 total subjects in metformin and placebo groups), the adverse events related to the metformin 
were similar to those seen in adults, including gastrointestinal symptoms (like diarrhea and abdominal pain) in $25 \%$ of participants [31]. No safety concerns were discovered regarding use of metformin in pediatric T2D patients.

In the TODAY study [30], only $48.3 \%$ of metforminonly treatment group were able to maintain glycemic control on the oral medication alone, supporting the notion that pediatric patients with T2D are more likely to require more pharmacologic therapies than adults [7]. Initial pharmacologic therapy should include metformin and/or insulin. For generally asymptomatic children over the age of 10 with an HbA1c of less than $8.5 \%$, providers should prescribe metformin twice a day (BID) [32]. Patients should begin on 500-1,000 mg metformin BID with food and titrate dosage by $500 \mathrm{mg}$, up to maximum dosage of 2,000 mg per day (as tolerated by the patient) [7, 14]. Insulin therapy should be started for patients unable to reach glycemic targets with oral metformin alone or who are unable to continue metformin treatment [14]. Adult doses of metformin are approved for use and are efficacious in youths over the age of 10 years. Metformin acts to increase peripheral insulin sensitivity and glucose uptake while decreasing glucose production in liver and gastrointestinal (GI) absorption [7]. Possible side effects of metformin therapy for children include GI disturbances, weight gain, anemia, elevated liver enzymes, and hypoglycemic episodes [33].

Initial treatment for symptomatic pediatric patients with an HbA1c greater than or equal to $8.5 \%$ or with blood glucose greater than or equal to $250 \mathrm{mg} / \mathrm{dL}$ should include metformin and basal insulin [14]. Recommended dosing for basal insulin is 0.5 units $/ \mathrm{kg} /$ day and can be increased every few days based on measured blood glucose levels [14]. The most common side effect of insulin is hypoglycemia and weight gain [34].
According to ADA guidelines, patients presenting with ketosis or diabetic ketoacidosis should be treated with subcutaneous or IV insulin immediately [7]. These patients should have their type of diabetes confirmed by lab testing (c-peptide, glucose, and auto-antibodies-after the glucose has been stabilized). If the patient has $\mathrm{T} 2 \mathrm{D}$, start metformin while overlapping with outpatient subcutaneous insulin [7]. Insulin may not be needed after the patient reaches stable glycemia [7, 14]. If a patient presents with a blood glucose greater than or equal to $600 \mathrm{mg} / \mathrm{dL}$, check for hyperglycemic hypertonic non-ketotic (HHNK) syndrome $[7,14]$.

If patients are unable to reach glycemic targets with metformin and basal insulin, the ADA recommends addressing medication adherence with the patient and family [7]. If still unable to reach targets, the ADA recommends providers consider "higher doses of long-acting insulin or initiation of multiple daily injections of basal and premeal rapid-acting insulin" [7]. Newly approved medications - not yet FDA-approved in children and not currently in ADA Standards of Care - may be useful for this patient population (Table 3) [35].

As of June 2019, a new subcutaneous agent, Liraglutide $\left(\right.$ Victoza $\left.{ }^{\circledR}\right)$, was approved for use in pediatric patients 10 years of age or older with T2D [45]. The 2020, ADA Standards of Care recommended that physicians consider adding Liraglutide if the patients has been unable to reach target glycemic control on initial pharmacologic agents [14]. Liraglutide is a GLP-1 receptor agonist (GLP-1RA) that functions to slow gastric emptying, decrease appetite, enhance postmeal insulin production, suppress glucagon secretion (and hence hepatic glucose production), and improve existing $\beta$-cell function to make more insulin [7]. Tamborlane et al. [46] reported that the one-year estimated treatment difference in hemoglobin A1c from liraglutide

Table 3: FDA-approved treatment for T2D in children.

\begin{tabular}{|c|c|c|}
\hline Medication class & Drugs & Minimum age for use \\
\hline Biguanides & Metformin & 10 years \\
\hline Glucagon-like peptide- 1 agonist & Liraglutide $\left(\right.$ Victoza $\left.^{\circledR}\right)$ [36] & 10 years \\
\hline \multirow[t]{3}{*}{ Insulins: Basal } & Glargine (Lantus) [37 $]^{\mathrm{a}}$ & 6 years \\
\hline & Detemir (Levemir) [38 $]^{\mathrm{a}}$ & 2 years \\
\hline & Degludec $\left(\right.$ Tresiba $\left.^{\circledR}\right)[39]^{\mathrm{a}}$ & 1 year \\
\hline \multirow[t]{5}{*}{ Insulin: Bolus/prandial } & Insulin NPH (Novolin N) $[40]^{a}$ & Not specified $(<12$ years not studied) \\
\hline & Insulin (Novolin R) [41] ${ }^{\mathrm{a}}$ & 2 years \\
\hline & Insulin Aspart (NovoLog $\left.{ }^{\circledR}\right)[42]^{\mathrm{a}}$ & 2 years \\
\hline & Insulin Lispro (Humalog) [43] ${ }^{\mathrm{a}}$ & 3 years \\
\hline & Insulin Glulisine (Apidra) [44] ${ }^{\mathrm{a}}$ & 4 years \\
\hline
\end{tabular}

Data taken from ADA Position Statement, FDA, and FDA Drug Labels [7, 35, 45]. Most of the insulin therapies have not been studied for safety and

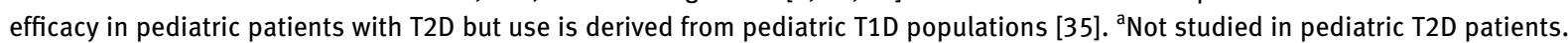


is $-1.30 \%$. The most common side effects include nausea, vomiting, and diarrhea; minor hypoglycemia is a less common side effect. Mean weight loss of $1.91 \mathrm{~kg}$ was also seen in the Liraglutide group at the end of the 52 weeks, whereas an average weight gain of $0.87 \mathrm{~kg}$ was measured in the placebo group [46]. Given the effectiveness of this medication, it may prove beneficial for pediatric patients unable to reach glycemic targets on metformin and insulin therapy alone.

It is important to note that most anti-diabetes medications approved for the management of T2D in adults are not FDA-approved for use in pediatric patients. Thus, their use cannot be recommended on a routine basis. The FDA notes many unique barriers to pediatric drug trials. Some of the more pronounced barriers include the ethical consideration of consent in children and the high potential for risks given possible differences in metabolism between adults and children [47]. In addition, safety concerns for pediatric participants have led the FDA to instill stricter guidelines for pediatric studies [47].

In terms of general classes of adult-approved medications for diabetes, sulfonylureas and meglitinides act by stimulating the patient's own pancreatic beta cells to secrete insulin. Sulfonylureas such as glimepiride have been studied in a small pediatric population and shown to lower HbA1c safely in children with T2D [48]. The most notable side effect compared to metformin in the trial was weight gain [48]. Other anti-diabetes medications available for the treatment of T2D in adults include alpha-glucosidase inhibitors, GLP-1RA, and DPP-4 inhibitors. Only the GLP-1RA class of medications has an FDA-approved medication (liraglutide) to be used in pediatric patients. Other options are amylin analogs that effect gastric emptying and glucagon secretion and SGLT-2 inhibitors that work in the kidneys to reduce reabsorption of glucose, both of which are not approved for use in the pediatric population.

Lastly, there are the thiazolidinediones, which perhaps have the most research available surrounding their use in pediatric patients with T2D based on the TODAY study [30] with rosiglitazone, but there are still no thiazolidinediones FDA approved for use in pediatric patients. In the TODAY study [30], metformin plus rosiglitazone treatment was compared to metformin alone and metformin with lifestyle interventions to see which had more durable glycemic control. That study found that rosiglitazone combined with metformin therapy lead to a larger percentage of participants maintaining glycemic control compared with the other two groups [30]. In terms of safety and tolerability, the study found comparable side effects and adverse effects between the three groups; however, subjects in the metformin plus rosiglitazone group did see significantly increased BMIs [33]. In addition, there are some clinical trials registered through clinicaltrials.gov investigating other pharmacologic options in pediatric patients with T2D, but the results on the effectiveness of the medication on glycemic control have not been published [49-54].

There are a number of ongoing clinical trials registered with clinicaltrials.gov that are evaluating the safety and efficacy of other adult FDA-approved T2D therapies in pediatric patients. Such studies include investigation of the safety and efficacy of Ertugliflozin (SGLT-2 inhibitor) (NCT04029480) [49], Dulaglutide (GLP-1RA) (NCT02963766) [50], and Dapagliflozin (SGLT-2 inhibitor) and Saxagliptin (DPP-4 inhibitor) (NCT03199053) [51]. Another study [52] is in phase 3 as of the writing of this review, researching the use of Alogliptin (DPP-4 inhibitor) (NCT02856113) in pediatric populations. Most notable of ongoing recruiting pediatric studies involving T2D are the Surgical or Medical Treatment (ST2OMP) trial [53] further investigating the efficacy of bariatric surgery in management of T2D in pediatric populations (NCT04128995) and the study [54] investigating effects bariatric surgery on T2D pancreatic, renal, and cardiovascular comorbidities in pediatric patients with T2D (NCT03620773). Future studies should be geared toward other promising diabetic medications not yet approved in the pediatric population.

While the 2020 American Diabetes Association Standards of Care [14] still recommended metformin and insulin as first line treatment of T2D, it is likely that the newer classes including GLP-1RA, DPP-4 inhibitors, and SGLT-2 inhibitors will be indicated for pediatric patients with $\mathrm{T} 2 \mathrm{D}$ in the future.

\section{Metabolic surgery}

ADA guidelines support metabolic surgery as a treatment option in some adolescent patients with T2D. Specifically, metabolic surgery may be beneficial in patients with a BMI $>35 \mathrm{~kg} / \mathrm{m}^{2}$ and comorbidities who are unable to reach glycemic stability with lifestyle and medications alone [7]. In order to ensure the safety of the patient and efficacy of the surgery, a full multidisciplinary team is required $[7,14]$.

Metabolic surgery is a relatively new treatment option for pediatric patients with T2D. In 2018, a secondary analysis [55] compared data from the 30 (out of 242) pediatric patients with T2D who underwent metabolic surgery as part of the Teen Longitudinal Assessment of Bariatric Surgery (Teen-LABS) study to 63 patients that were matched by similar baseline characteristics from the TODAY study (pharmacologic and lifestyle therapy in youths with T2D). Mean A1c decreased from 6.8\% at 
baseline to $5.5 \%$ at two years post-surgery in the TeenLABS cohort, whereas in the TODAY cohort, there was a mean A1c increase from $6.4 \%$ at baseline to $7.8 \%$ at two years postsurgery [55]. Other notable results postmetabolic surgery included decreases in waist circumference $(-19.2 \%$ Teen-LABS cohort; $+3.55 \%$ TODAY cohort), an overall decrease in BMI $(-29.2 \%$ Teen-LABS cohort; $+3.5 \%$ TODAY cohort), and reduction in triglyceride levels $(-19 \%$ Teen-LABS cohort; $+15.3 \%$ TODAY cohort) at the 2-year follow up compared to starting baseline levels, demonstrated in Table 4 [55]. More research is still needed to determine long-term effects of metabolic surgery [7]; however, a 2019 study [56] reported that metabolic surgery is as safe and effective in adolescents as in adult-based populations, further supporting its validity as a treatment option.

\section{Complications}

Youth-onset T2D is associated with a higher risk of complications and mortality than adult-onset T2D [57]. Similar complications are seen, but youths with T2D develop complications earlier than those with adult-onset T2D [6]. In addition, a study [6] comparing onset of complications in youths with T2D vs those with T1D showed complications occurring sooner in pediatric patients diagnosed with T2D. Specifically, earlier renal and neurologic complications were reported, but no significant change in time course for retinopathy was observed [6].

For perspective, in the TODAY study, the prevalence of hypertension was $11.6 \%$ at baseline (where mean age was 14 years) and rose to $33.8 \%$ by the study's end [58]. In addition, high-risk LDL levels rose from 4.5 (baseline) to $10.7 \%$ (study's end), evidence of microalbuminuria rose from 6.3 (baseline) to $16.6 \%$ (study's end), and 6.3\% had microalbuminuria at baseline compared to $16.6 \%$ at the end of the study [58]. The prevalence of diabetic retinopathy at the end of the TODAY study was 13.9\% [58]. Studies also found that young patients were less likely to be treated

Table 4: Significant comparisons between Teen-LABS and TODAY cohorts.

\begin{tabular}{lrr}
\hline Outcomes & Teen-LABS & TODAY \\
\hline HbA1c\% & $-19.1 \%(6.8 \rightarrow 5.5)$ & $+21.9 \%$ \\
Average body mass index & $-29.2 \%$ & $+7.8)$ \\
Waist circumference & $-19.2 \%$ & $+3.5 \%$ \\
Triglyceride levels & $-19 \%$ & $+15.3 \%$ \\
\hline
\end{tabular}

Values are shown as percent difference in least-squares means after two years as a percentage of the baseline value [55]. for hypertension and dyslipidemia, further exacerbating the severity of complications [57].

In another study [59] looking at the prevalence of impaired glucose tolerance (prediabetes or T2D) in youth with nonalcoholic fatty liver disease (NAFLD), an estimated $23.4 \%$ of participants had prediabetes and $6.5 \%$ T2D. NAFLD associated with the pediatric population appears more aggressive compared to adult NAFLD with more severe histologic changes. This increase in severity is associated with a higher risk of progressing to liver complications including fibrosis, cirrhosis, and hepatic failure $[7,59]$.

Luckily, screening and management for T2DM complications/comorbidities in pediatric populations is very similar to screening methods in adults. The ADA-recommended screening modalities and primary treatment options are summarized in Table 5 [7]. More research is still needed into many of the complications associated with pediatric-specific populations.

\section{Technology to support children with type 2 diabetes}

There were substantial advances during the decade covered in this review in the measurement of glucose and insulin delivery [61]. While tools such as continuous glucose monitoring, insulin pumps, and hybrid closed loop systems have improved time in range, reduced hypoglycemia rates, and improved patients' quality of life, their use in T2D is less common due to a lack of insurance coverage [62].

Children who are on three or more insulin injections per day and doing four or more fingerstick glucose checks per day are most likely to benefit from a continuous glucose monitor or an insulin pump. However, it is likely that these tools will be used more widely in the future and may benefit children with T2D as well. Excellent resources are available online to demonstrate the range of technology to support diabetes care [63].

\section{Osteopathic considerations}

The benefits of an osteopathic approach to T2D in adults has been well established and can readily be applied to pediatric patients [64]. The first tenet of osteopathy states that the body is a unit, and the person is a unit of body, mind, and spirit [65]. Osteopathic physicians should readily account for full spectrum of factors, including the 
Table 5: Summary of complication screening with modality and primary treatment options based on ADA recommendations [7, 14].

\begin{tabular}{|c|c|}
\hline Complication & Management \\
\hline Nephropathy & $\begin{array}{l}\text { Monitor with regular blood pressure screenings at each visit. If high, treat with lifestyle interventions, if still } \\
\text { high after six months then start ACE inhibitor/ARB therapy [7]. } \\
\text { Obtain spot UACR, eGFR (calculated from serum creatinine and height with Schwartz equation), and serum } \\
\text { potassium levels. If high, begin ACE inhibitor/ARB therapy (confirm elevated UACR on } 2 \text { of } 3 \text { samples) [7]. }\end{array}$ \\
\hline Neuropathy & Inspect feet, pulses, 5-g monofilament test, vibration sensations, and ankle reflexes [7]. \\
\hline Retinopathy & Dilated eye exam at optometrist or ophthmalogist [7]. \\
\hline Non-alcoholic fatty liver disease & $\begin{array}{l}\text { Screen with AST and ALT levels. If high, treat with weight loss regimen and consider referral to gastro- } \\
\text { enterology unable to control [7]. }\end{array}$ \\
\hline Obstructive sleep apnea & $\begin{array}{l}\text { Screen for symptoms and refer to specialist for sleep study if needed [7]. Possible signs and symptoms } \\
\text { include audible pauses in breathing while sleeping, snoring, restless sleep, inattentiveness at school, and } \\
\text { behavioral abnormities. Excessive sleepiness is not common as is typical in adults [60]. }\end{array}$ \\
\hline Polycystic ovarian syndrome & $\begin{array}{l}\text { Screen for signs and symptoms and possible laboratory evaluation. Treat with metformin and lifestyle } \\
\text { modifications, can consider oral contraceptives [7]. }\end{array}$ \\
\hline Cardiovascular disease & $\begin{array}{l}\text { Testing not recommended without symptoms, focus on lifestyle modifications and obtaining glycemic } \\
\text { control [7]. }\end{array}$ \\
\hline Dyslipidemia & $\begin{array}{l}\text { Lipid panel after initial glycemic control. If abnormal lipid levels, encourage/support diet modifications and } \\
\text { glycemic control for } 6 \text { months, if unable to improve, consider statin therapy. If severe hypertriglyceridemia, } \\
\text { consider fibrates + controlling blood sugar to due pancreatitis risk, noting use of fibrates in this population } \\
\text { has not been studied and use is extrapolated from adults [7]. }\end{array}$ \\
\hline
\end{tabular}

psychosocial factors discussed in this review, to allow for a holistic approach to pediatric patients with T2D [64].

The second tenet of osteopathy - the body is capable of self-regulation, self-healing, and health maintenance - is applicable toward treatment plans in this population [65]. Physicians have a unique opportunity to empower and educate patients and families on the importance of selfcare involved in diabetes management [64]. Importantly, the barriers and circumstances of the parents/caregivers should be considered in terms of ability to teach and encourage self-care in their children.

The third tenet of osteopathy states that structure and function are reciprocally interrelated [65]. The recommendations that physicians can give regarding physical activity will improve insulin sensitivity in muscles, which can improve glycemic control [64].

The fourth tenet of osteopathy states that rational treatment is based upon an understanding of the basic principles of body unity, self-regulation, and the interrelationship of structure and function [65]. Providers should use their knowledge of osteopathy to provide patient-centered care and promote health in youth presenting with T2D.

\section{Study limitations}

This study has a number of limitations. This review is intended to be a clinically-focused narrative review; it is not intended to be comprehensive of all literature sources or a systematic review and meta-analysis. It is possible that there are additional studies that were not reported in this manuscript. In our review, we only included studies found in the databases used for our search, or those referenced in our current sources.

\section{Conclusions}

The prevalence of T2D is increasing among children. Further, this condition is more virulent, progresses more rapidly, and is less responsive to treatment than when it is diagnosed in adults. To address these challenges, physicians should screen those at risk, diagnose early, and use a comprehensive family-based approach to address these serious chronic and progressive condition.

Research funding: None reported.

Author contributions: Ms. Molinari provided substantial contributions to conception and design, acquisition of data, or analysis and interpretation of data; Ms. Molinari drafted the article and Dr. Shubrook revised it critically for important intellectual content; both authors gave final approval of the version of the article to be published; and both authors agree to be accountable for all aspects of the work in ensuring that questions related to the accuracy or integrity of any part of the work are appropriately investigated and resolved.

Competing interests: Dr. Shubrook has served on advisory boards for Sanofi, NovoNordisk, EliLilly, MannKind, and Bayer. 
Disclaimer: Dr. Shubrook, a former JAOA Associate Editor, was not involved in the editorial review or decision to publish this article.

\section{References}

1. National Diabetes Statistics Report. Estimates of diabetes and its burden in the United States. Centers for Disease Control and Prevention Website; 2020. https://www.cdc.gov/diabetes/pdfs/ data/statistics/national-diabetes-statistics-report.pdf [Accessed 7 May 2020].

2. Mayer-Davis EJ, Lawrence JM, Dabelea D, Divers J, Isom S, Dolan L, et al. Incidence trends of type 1 and type 2 diabetes among youths, 2002-2012. N Engl J Med 2017;376:1419-29.

3. The RISE Consortium. Metabolic contrasts between youth and adults with impaired glucose tolerance or recently diagnosed type 2 diabetes: I. Observations using the hyperglycemic clamp. Diabetes Care 2018;41:1696-706.

4. The RISE Consortium. Metabolic contrasts between youth and adults with impaired glucose tolerance or recently diagnosed type 2 diabetes: Il. Observations using the oral glucose tolerance test. Diabetes Care 2018;41:1707-16.

5. Copeland KC, Zeitler P, Geffner M, Guandalini C, Higgins J, Hirst K, et al. Characteristics of adolescents and youth with recent-onset type 2 diabetes: the TODAY cohort at baseline. J Clin Endocrinol Metab 2011;96:159-67.

6. Dart AB, Martens PJ, Rigatto C, Brownell MD, Dean HJ, Sellers EA. Earlier onset of complications in youth with type 2 diabetes. Diabetes Care 2014;37:436-43.

7. Arslanian S, Bacha F, Grey M, Marcus MD, White NH, Zeitler P. Evaluation and management of youth-onset type 2 diabetes: a position statement by the American Diabetes Association. Diabetes Care 2018;41:2648-68.

8. The TODAY Study Group. Treatment options for type 2 diabetes in adolescents and youth: a study of the comparative efficacy of metformin alone or in combination with rosiglitazone or lifestyle intervention in adolescents with type 2 diabetes. Pediatr Diabetes 2007;8:74-87.

9. Goran MI, Gower BA. Longitudinal study on Pubertal insulin resistance. Diabetes 2001;50:2444-50.

10. Cree-Green M, Triolo TM, Nadeau KJ. Etiology of insulin resistance in youth with type 2 diabetes. Curr Diabetes Rep 2013;13:81-8.

11. DeFronzo RA. From the triumvirate to the ominous octet: a new paradigm for the treatment of type 2 diabetes mellitus. Diabetes 2009;58:773-95.

12. Hales CM. Prevalence of obesity among adults and youth: United States, 2015-2016. NCHS Data Brief 2017;288:8.

13. Hardy OT, Czech MP, Corvera S. What causes the insulin resistance underlying obesity? Curr Opin Endocrinol Diabetes Obes 2012;19:81-7.

14. American Diabetes Association. Standards of medical Care in Diabetes-2020. Diabetes Care 2020;43:S77-88.

15. Klingensmith G), Pyle L, Arslanian S, Copeland K, Cuttler L, Kaufman $F$. The presence of GAD and IA-2 antibodies in youth with a type 2 diabetes phenotype: results from the TODAY study. Diabetes Care 2010;33:1970-5.
16. Brar PC. Update on the current modalities used to screen high risk youth for prediabetes and/or type 2 diabetes mellitus. Ann Pediatr Endocrinol Metab 2019;24:71-7.

17. Nowicka P, Santoro N, Liu H, Lartaud D, Shaw M, Goldberg R, et al. Utility of Hemoglobin A1c for diagnosing prediabetes and diabetes in obese children and adolescents. Diabetes Care 2011; 34:1306-11.

18. Compas BE, Jaser SS, Dunn MJ, Rodriguez EM. Coping with chronic illness in childhood and adolescence. Annu Rev Clin Psychol 2012;8:455-80.

19. Peters A, Laffel L, The American Diabetes Association Transitions Working Group. Diabetes care for emerging adults: recommendations for transition from pediatric to adult diabetes care systems: a position statement of the American diabetes association. Diabetes Care 2011;34:2477-85.

20. Anderson BJ, Edelstein S, Abramson NW, Levitt Katz LE, Yasuda PM, Lavietes SJ, et al. Depressive symptoms and quality of life in adolescents with type 2 diabetes: baseline data from the TODAY study. Diabetes Care 2011;34:2205-7.

21. Mojtabai R, Olfson M, Han B. National trends in the prevalence and treatment of depression in adolescents and young adults. Pediatrics 2016;138:e20161878.

22. The TODAY Study Group. Binge eating, mood, and quality of life in youth with type 2 diabetes: baseline data from the TODAY study. Diabetes Care 2011;34:858-60.

23. Galuska DA, Gunn JP, O'Connor AE, Petersen R. Addressing childhood obesity for type 2 diabetes prevention: challenges and opportunities. Diabetes Spectr 2018;31:330-5.

24. Pulgaron ER, Delamater AM. Obesity and type 2 diabetes in children: epidemiology and treatment. Curr Diabetes Rep 2014; 14:508.

25. Grossman DC, Bibbins-Domingo K, Curry SJ, Barry MJ, Davidson KW, Doubeni CA, et al. US Preventive Services Task Force. Screening for obesity in children and adolescents: US preventive services task force recommendation statement. JAMA 2017;317:2417.

26. Hales CM, Fryar CD, Carroll MD, Freedman DS, Ogden CL. Trends in obesity and severe obesity prevalence in us youth and adults by sex and age, 2007-2008 to 2015-2016. JAMA 2018;319:1723.

27. Andes LJ, Cheng YJ, Rolka DB, Gregg EW, Imperatore G. Prevalence of prediabetes among adolescents and young adults in the United States, 2005-2016. JAMA Pediatr 2020;174:e194498.

28. Magge SN, Silverstein J, Elder D, Nadeau K, Hannon TS. Evaluation and treatment of prediabetes in youth. J Pediatr 2020; 219:11-22.

29. The RISE Consortium. Impact of insulin and metformin versus metformin alone on $\beta$-cell function in youth with impaired glucose tolerance or recently diagnosed type 2 diabetes. Diabetes Care 2018;41:1717-25.

30. TODAY Study Group. A clinical trial to maintain glycemic control in youth with type 2 diabetes. N Engl J Med 2012;366:2247-56.

31. Jones KL, Arslanian S, Peterokova VA, Park J-S, Tomlinson MJ. Effect of metformin in pediatric patients with type 2 diabetes: a randomized controlled trial. Diabetes Care 2002;25:89-94.

32. Riomet ER (metformin) [package insert]. U.S. Food and Drug Administration website; Revised August 2019. Available from: https://www.accessdata.fda.gov/drugsatfda_docs/label/2019/ 212595s000lbl.pdf. [Accessed 1 June 2020].

33. TODAY Study Group. Safety and tolerability of the treatment of youth-onset type 2 diabetes: the TODAY experience. Diabetes Care 2013;36:1765-71. 
34. Diabetes.org. Hypoglycemia (low blood glucose). American Diabetes Association. Available from: http://www.diabetes.org/ living-with-diabetes/treatment-and-care/blood-glucosecontrol/hypoglycemia-low-blood.html [Accessed 3 Aug 2019].

35. Gourgari E, Wilhelm EE, Hassanzadeh H, Aroda VR, Shoulson I. A comprehensive review of the FDA-approved labels of diabetes drugs: indications, safety, and emerging cardiovascular safety data. J Diabet Complicat 2017;31:1719-27.

36. Victoza ${ }^{\circledR}$ (Liraglutide) [package insert]. Novo Nordisk, Plainsboro, NJ; Revised June 2019. Available from: https://www.novo-pi. com/victoza.pdf [Accessed 20 May 2020].

37. Lantus (glargine) [package insert]. U.S. Food and Drug Administration Website; Revised June 2009. Available from: https://www.accessdata.fda.gov/drugsatfda_docs/label/2009/ 021081s034lbl.pdf [Accessed 20 May 2020].

38. Levemir $^{\circledR}$ (detemir) [package insert]. U.S. Food and Drug Administration Website. Revised January 2019. Available from: https://www.accessdata.fda.gov/drugsatfda_docs/label/2019/ 021536s054lbl.pdf [Accessed 1 June 2020].

39. Tresiba $^{\circledR}$ (insulin degludec) [package insert]. Novo Nordisk, Plainsboro, NJ; Revised November 2019. Available from: https:// www.novo-pi.com/tresiba.pdf [Accessed 20 May 2020].

40. Novolin ${ }^{\circledR}$ N (insulin NPH) [package insert]. U.S. Food and Drug Administration Website; Revised November 2019. Available from: https://www.accessdata.fda.gov/drugsatfda_docs/label/2019/ 019959s082lbl.pdf [Accessed 1 June 2020].

41. Novolin ${ }^{\circledR} R$ (regular, human insulin USP) [package insert]. U.S. Food and Drug Administration Website, Princeton, N]; Revised February 2012. Available from: https://www.accessdata.fda.gov/drugsatfda_ docs/label/2012/019938s066lbl.pdf [Accessed 1 June 2020].

42. NovoLog ${ }^{\circledR}$ (insulin aspart) [package insert]. U.S. Food and Drug Administration Website, Plainsboro, NJ; Revised February 2015. Available from. https://www.accessdata.fda.gov/ drugsatfda_docs/label/2015/020986s082lbl.pdf [Accessed 1 June 2020].

43. Humalog (insulin lispro) [package insert]. U.S. Food and Drug Administration Website, Indianapolis, IN; Revised March 2013. Available from: https://www.accessdata.fda.gov/drugsatfda_ docs/label/2013/020563s115lbl.pdf [Accessed 1 June 2020].

44. Apidra (insulin glulisine) [package insert]. U.S. Food and Drug Administration Website, Bridgewater, NJ; Revised October 2008. Available from: https://www.accessdata.fda.gov/drugsatfda_ docs/label/2008/021629s015lbl.pdf [Accessed 1 June 2020].

45. FDA approves new treatment for pediatric patients with type 2 diabetes. FDA; 2019. Available from: http://www.fda.gov/newsevents/press-announcements/fda-approves-new-treatmentpediatric-patients-type-2-diabetes [Accessed 22 July 2019].

46. Tamborlane WV, Barrientos-Pérez M, Fainberg U, Frimer-Larsen $\mathrm{H}$, Hafez M, Hale PM, et al. Liraglutide in children and adolescents with type 2 diabetes. N Engl J Med 2019;381:637-46.

47. Center for DrugEvaluation and Research. Drug research and children. U.S. Food and Drug Administration. Available from: https://www.fda.gov/drugs/drug-information-consumers/drugresearch-and-children [Accessed 29 July 2020].

48. Gottschalk M, Danne T, Vlajnic A, Cara JF. Glimepiride versus metformin as monotherapy in pediatric patients with type 2 diabetes: a randomized, single-blind comparative study. Diabetes Care 2007;30:790-4.
49. Ertugliflozin type 2 diabetes mellitus (T2DM) pediatric study (MK8835/PF-04971729) (MK-8835-059). ClinicalTrials.gov. Available from: https://clinicaltrials.gov/ct2/show/NCT04029480 [Accessed 2 Aug 2020].

50. A study of dulaglutide (LY2189265) in children and adolescents with type 2 diabetes. Clinicaltrials.gov; 2020. Available from: https://clinicaltrials.gov/ct2/show/NCT02963766 [Accessed 31 July 2020].

51. Study to evaluate safety and efficacy of dapagliflozin and saxagliptin in patients with type 2 diabetes mellitus aged 10 to below 18 years old. Clinicaltrials.gov; 2020. Available from: https://clinicaltrials.gov/ct2/show/NCT03199053 [Accessed 31 July 2020].

52. Phase 3 alogliptin pediatric study. Clinicaltrials.gov. Available from: https://clinicaltrials.gov/ct2/show/NCT0285611 [Accessed 2 Aug 2020].

53. Surgical or medical treatment. Clinicaltrials.gov. Available from: https:// clinicaltrials.gov/ct2/show/NCT04128995 [Accessed 2 Aug 2020].

54. Bjornstad P. Impact of metabolic surgery on pancreatic, renal and cardiovascular health in youth with type 2 diabetes. Clinicaltrials.gov; 2020. Available from: https://clinicaltrials. gov/ct2/show/NCT03620773 [Accessed 31 July 2020].

55. Inge TH, Laffel LM, Jenkins TM, Marcus MD, Leibel NI, Brandt ML, et al. Comparison of surgical and medical therapy for type 2 diabetes in severely obese adolescents. JAMA Pediatr 2018;172:452.

56. Bolling CF, Armstrong SC, Reichard KW, Michalsky MP. Metabolic and bariatric surgery for pediatric patients with severe obesity. Pediatrics 2019;144:e20193224.

57. Al-Saeed AH, Constantino MI, Molyneaux L, D’Souza M, Limacher-Gisler F, Luo C, et al. An inverse relationship between age of type 2 diabetes onset and complication risk and mortality: the impact of youth-onset type 2 diabetes. Diabetes Care 2016;39:823-9.

58. Tryggestad JB, Willi SM. Complications and comorbidities of T2DM in adolescents: findings from the TODAY clinical trial. J Diabet Complicat 2015;29:307-12.

59. Newton KP, Hou J, Crimmins NA, Lavine JE, Barlow SE, Xanthakos $\mathrm{SA}$, et al. Prevalence of prediabetes and type 2 diabetes in children with nonalcoholic fatty liver disease. JAMA Pediatr 2016; 170:e161971.

60. Kansagra S, Vaughn B. Pediatric sleep apnea: five things you might not know. Neurol Clin Pract 2013;3:321-5.

61. Sherr JL. Closing the loop on managing youth with type 1 diabetes: children are not just small adults. Diabetes Care 2018; 41:1572-8.

62. Juvenile Diabetes Research Foundation Continuous Glucose Monitoring Study Group. Effectiveness of continuous glucose monitoring in a clinical care environment: evidence from the juvenile diabetes research foundation continuous glucose monitoring (JDRF-CGM) trial. Diabetes Care 2010;33:17-22.

63. Diabetes Wise. Diabetes wise. Available from: https:// diabeteswise.org [Accessed 25 Oct 2020].

64. Shubrook JH, Jr, Johnson AW. An osteopathic approach to type 2 diabetes mellitus. J Am Osteopath Assoc 2011;111:531-7.

65. Tenets of Osteopathic Medicine. American Osteopathic Association. Available from: https://osteopathic.org/about/ leadership/aoa-governance-documents/tenets-of-osteopathicmedicine/ [Accessed 1 Sept 2019]. 